\title{
CAMBIO EN LAS TEORÍAS CIENTíFICAS DESDE LA VISIÓN DE ESTUDIANTES DE EDUCACIÓN EN LA UNIVERSIDAD DE LOS ANDES, VENEZUELA
}

\section{CHANGE IN SCIENTIFIC THEORIES FROM THE POINT OF VIEW OF EDUCATION STUDENTS AT THE ANDES UNIVERSITY, VENEZUELA}

\author{
José Escalona Tapia ${ }^{1}$, Bernardo Fontal Rivera ${ }^{2}$ \\ ${ }^{1}$ Doctor en Ciencias de la Educación, profesor asociado de la Facultad de Humanidades y Educación. Universidad de Los \\ Andes, Mérida, Venezuela, e-mail: cieduc@ula.ve; ${ }^{2}$ Doctor en Química, profesor titular (jubilado) de la Facultad de Ciencias. \\ Universidad de Los Andes, Mérida, Venezuela, e-mail: bfontal@ula.ve
}

Rev. U.D.C.A Act. \& Div. Cient. 19(2): 467-477, Julio-Diciembre, 2016

\section{RESUMEN}

Las concepciones son la base fundamental en el proceso educativo, con el fin de planificar acciones didácticas, necesarias para lograr cambios graduales, hacia una visión integrada de la naturaleza científica. Por ello, este trabajo estudió el cambio de concepciones sobre teorías científicas en estudiantes de educación de la Universidad de Los Andes. El enfoque de investigación-acción exigió una metodología cualitativa y descriptiva, para evaluar la incidencia de un curso con carácter progresista en la modificación de concepciones sobre teorías científicas. Las conclusiones mostraron que el curso fue importante para modificar algunas concepciones sobre las teorías científicas; las concepciones que más destacan son las positivistas; las clases apuntaladas con video resultaron ser las de mayor dinámica y discusión sobre las ciencias.

Palabras clave: Cambio de concepciones, evaluación, curso progresista, teorías científicas.

\section{SUMMARY}

Conceptions are fundamental to plan didactic actions in the achievement of gradual changes towards an integrated vision of the science. This investigation evidenced the change of conceptions in scientific theories in students of education of the University of The Andes, Venezuela. The methodology was descriptive to evaluate a course with progressive character in the modification of conceptions on the scientific theories. The conclusions show that: the course was important to modify some conceptions on the scientific theories; the conceptions that more stand out are positivists; the classes supported with video turned out to be of major dynamics and discussion on the sciences.

Key words: Change conceptions, evaluation, progressive course, scientific theories.

\section{INTRODUCCIÓN}

Diversas experiencias universitarias y publicaciones patentizan que el positivismo y el experimentalismo han dominado el escenario científico contemporáneo. Tal tendencia ha conducido a diversas pesquisas, de cómo las creencias científicas pueden influir en la comprensión fenomenológica de la ciencia y en la formación didáctica del profesorado. $Y$ es que sí se entiende al conocimiento cultural como un patrimonio de representaciones de la realidad, obtenido desde teorizaciones personales; también es lógico pensar que quien aprende ciencias se verá influenciado por los métodos usados para su aprendizaje y el propio contexto educativo.

Por ello, se ha señalado que muchas concepciones erróneas sobre las ciencias se originan en las clases iniciales, pudiendo afianzarse durante el resto del proceso educativo o al entrar en contracción con otras ideas posteriores (Pontes et al. 2015). Aunado a ello, se ha observado que los docentes no siempre están bien preparados para entender y hacer entender la enseñanza científica y, por ello, las propias experiencias de aprendizaje, los estereotipos y los materiales de enseñanza median, decisivamente, las concepciones profesorales sobre la ciencia.

Se sabe que lo trasmitido, a través de los medios de información de masas, impregna el lenguaje de la enseñanza científi- 
ca cotidiana y, en general, a la educación, formando concepciones erradas en las diferentes generaciones profesorales de las ciencias (Serrano, 2015). Por ello, Fourez (2008), al referirse a las concepciones y el lenguaje en el aprendizaje, indica que el ideario de cada persona permite el afloramiento de estructuras de comprensión y de explicación.

También, se sabe que para la formación del profesorado han emergido diversos compendios, que explicitan que las estrategias didácticas docentes varían en cada cátedra, que cada asignatura tiene sus propias creencias sobre la enseñanza y el aprendizaje y que se suele creer que el conocimiento disciplinar es suficiente para desarrollar destreza docente.

Por otro lado, la mayoría de autores de hoy tienden a entender que no se debe admitir la existencia de un acuerdo único de reglas metodológicas para describir/explicar los postulados científicos, sino la emergencia de un armado continuo y dinámico con diversos enfoques de pensamiento; algunos le llaman enfoque continuo histórico integral.

Por lo anterior, resulta importante poder realizar clasificaciones sobre las corrientes de pensamiento, que puedan exponer los estudiantes sobre el andamiaje de las ciencias. Es verdad que no siempre se pueden sistematizar las respuestas en una tendencia u otra, pero, según diversos estudios epistémicos, tales ideas se pueden llegar a clasificar como:

Ideas Positivistas, donde se incluye la observación, la demostración y la descripción experimental de una realidad, mediante hipótesis o expresiones teóricas, basadas en verdades absolutas y el método científico, con inclinación hacia el entendimiento de las teorías, como explicaciones del funcionamiento de los fenómenos en el universo y las leyes, como reglas de la fenomenología científica.

Ideas Transicionales, las que toman en cuenta el progreso de la ciencia, mediante una evolución dada por un conocimiento científico que no está claro o no se ha establecido absolutamente, dejando ver un "alternativismo", donde prima la comprensión parcial de la ciencia moldeada por modelos, donde una de varias opciones son elegibles y competitivismo, donde varias opciones, si ser rivales, compiten por ofrecer mejores explicaciones.

Ideas Lakatosianas, las cuales, indican un progreso científico orientado por un conflicto teórico, generado entre grupos o comunidades de científicos, quienes exponen posiciones encontradas; resaltan la contradicción entre programas de investigación, que requieren la elaboración de hipótesis rivales y su evaluación, a través de nuevas pruebas científicas.

El objetivo de este trabajo fue valorar la incidencia del curso denominado "Estructura de la Materia", ubicada en el III semestre y desarrollado con enfoque progresista de la ciencia, para la modificación de concepciones epistemológicas sobre las teorías científicas, en estudiantes de Licenciatura en Educación, de la Universidad de Los Andes, en Mérida, Venezuela.

\section{METODOLOGÍA}

Los materiales usados tuvieron que ver con el desarrollo didáctico del curso; se usaron computadoras, proyectores de video, diversidad de sustancias químicas, variado instrumental de laboratorio, guías de prácticas y textos de carácter histórico sobre la ciencia; para la investigación, se empleó una grabadora de audio, para el registro de debates y de clases.

La investigación refiere concepciones de los participantes de un modo directo, por lo que fue cualitativa con carácter exploratorio-descriptivo de campo, presentando sistematizaciones de frecuencias y combinando descripciones cualitativas y cuantitativas (Schettini \& Cortazzo, 2015). Dado que posibilitó el diagnóstico, propuesta y estudio sobre el cambio en una determinada situación educativa, fue una investigación-acción (Schettini \& Cortazzo, 2015). El diagnóstico permitió desarrollar la base epistemológica general del estudiantado y, con ello, se elaboró una propuesta, que involucró la transformación del programa de la asignatura denominada "estructura de la materia" y la inclusión de tres modalidades de trabajo, como fueron: trabajo práctico con desarrollo de experiencias de laboratorio, con su consecuente ubicación en cada contexto histórico y el debate sobre los principios, las leyes o las teorías que sustentaban cada uno de los experimentos; sesiones de video, donde se eligieron representaciones de corte histórico y que hicieran alusión a los diversos caminos que ha tenido el conocimiento de la materia en variadas épocas históricas y sesiones de debate, en las que se retomaban la realización de experimentos y la discusión de los videos, a fin de establecer el camino temporal, sustentado cada conocimiento sobre la materias y sus implicaciones en la vida humana. La selección de los participantes fue intencional, participando 79 estudiantes, quienes iniciaron y finalizaron un curso denominado "Estructura de la Materia", ubicado en el ciclo inicial de la Mención Ciencias Físico-Naturales, de la Escuela de Educación, de la Universidad de Los Andes, Mérida, Venezuela.

La investigación, se llevó a cabo durante cuatro semestres consecutivos, con estudiantes que asistieron a las clases de un mismo docente, para estudiar el impacto del curso predeterminado; usándose la observación participante, mediante una guía de observación abierta y grabación de audio, para recoger las acciones cotidianas de los sujetos estudiados y acopiar descripciones necesarias para la investigación. Para la recolección de la información desde los estudiantes, se usó el cuestionario abierto, para referenciar directamente las 
opiniones de los sujetos en estudio al inicio y final del cada semestre, aplicándose una pregunta abierta, redactada del siguiente modo: "Una vez que los científicos han desarrollado una teoría, como, por ejemplo, la teoría atómica ¿La teoría podría sufrir cambios? Si usted cree que las teorías pueden cambiar o mantenerse iguales, explique ¿ipor qué debemos aprender sobre ellas? Defienda su respuesta con ejemplos". Las respuestas dadas por los estudiantes fueron organizadas textualmente en cuadros para cada semestre y, desde ellas, se extrajeron las categorías y sub-categorías de cada caso, para hacer un compendio de ellas y proceder a clasificarlas como positivistas, transiocionales o lakatosianas. Se debe destacar que a lo largo del estudio, se originaron ocho cuadros, que contenían las categorías y sub-categorías de los test iniciales y finales, los cuales, se resumen en los tres cuadros presentados en esta investigación. Del mismo modo, luego de cada encuentro con los estudiantes, se escuchaban las grabaciones sobre los debates y se extraían los indicadores generados desde las preguntas orientadoras, que giraban en torno al cambio o no de los fundamentos científicos en cada momento histórico vivido por la humanidad.

Por otra parte, se debe mencionar que la validez de este estudio pasa por reconocer que hace referencia directa a lo que responden los sujetos de estudio en función de una pregunta clara y concreta. Por ello, las respuestas se constituyen en un esbozo de lo que piensa cada estudiante al llegar al curso y cómo esa idea sobre "las teorías en ciencias" puede cambiar una vez que ha participado del curso y de las acciones metodológicas, en él incluidas. Así, las respuestas son una reseña directa del andamiaje epistemológico que presente el estudiante antes y después de estar en el curso y, por tanto, la calcificación como respuesta positivista, transicional o lakatosiana, en una inferencia totalmente adecuada, para todo el análisis cualitativo realizado.
El carácter "progresista" del curso fue definido partiendo de la construcción del conocimiento con incidencia histórica, no lineal de la ciencia y admitiendo la presencia del conflicto, la competencia y el error, como elementos de trabajo. Esto quiere decir que, para el caso del trabajo de laboratorio, por ejemplo, se planteaban las ideas iniciales sobre el fenómeno a modo de hipótesis; se realizaba el experimento; se lo ubicaba históricamente desde sus primeras realizaciones y con los cambios que ha tolerado hasta momento $y$, finalmente, se comparaban los contextos del conocimiento sobre el tema del experimento antes y después de su aparición en el andamiaje epistemológico de la ciencia, tratando de dejar siempre claro las nuevas metodologías de trabajo, otras formas de pensar, la evaluación de errores anteriores y la presencia de nueva instrumentación.

\section{RESULTADOS Y DISCUSIÓN}

Al analizar los cuadros 1 y 2 , se evidencia que la tendencia al inicio de cada curso fue la prevalencia de las concepciones positivistas, lo cual, coincide con varios estudios que reportan que las ideas del conocimiento científico se acercan a un discernimiento acabado y tomado por verdadero (Restrepo, 2014); esto debe ser considerado de importancia, por cuanto la finalidad educativa científica para la formación de una nueva naturaleza de la ciencia pasa por valorar la necesidad de un profesorado con otras ideas sobre las ciencias.

Se debe hacer notar que la mayor parte del grupo estudiado estuvo de acuerdo en que las teorías sí pueden cambiar en razón de detalles experimentales y demostraciones, por fallos, por no poseer bases científicas suficientes, por la búsqueda de conocimientos e investigación o porque cambian los métodos. Tal tendencia coincide con la conocida idea denominada axiomatización de las teorías, donde se aspira

Cuadro 1. Registra la clasificación de frecuencia para las concepciones positivistas, transicionales, Lakatosianas y otras, encontradas en el pre y post- test, para la pregunta de investigación realizada a estudiantes del curso "Estructura de la Materia", de la Escuela de Educación, de la Universidad de Los Andes, Venezuela.

\begin{tabular}{|c|c|c|c|c|c|c|c|c|}
\hline Semestre & \multicolumn{2}{|c|}{ SEMESTRE 1} & \multicolumn{2}{|c|}{ SEMESTRE 2} & \multicolumn{2}{|c|}{ SEMESTRE 3} & \multicolumn{2}{|c|}{ SEMESTRE 4} \\
\hline Concepción & Pre.T & Pos.T & Pre.T & Pos.T & Pre.T & Pos.T & Pre.T & Pos.T \\
\hline Positivista & $7 / 17$ & $9 / 9$ & $9 / 19$ & $7 / 12$ & $6 / 24$ & $9 / 16$ & $10 / 19$ & $8 / 10$ \\
\hline Totales & \multicolumn{4}{|c|}{ 32/79 PRE-TEST } & \multicolumn{4}{|c|}{ 33/47 POST-TEST } \\
\hline Transicional & $4 / 17$ & $5 / 9$ & $3 / 19$ & $6 / 12$ & $3 / 24$ & $2 / 16$ & $2 / 19$ & $2 / 10$ \\
\hline Totales & \multicolumn{4}{|c|}{ 12/79 PRE-TEST } & \multicolumn{4}{|c|}{ 15/47 POST-TEST } \\
\hline Lakatosiana & $2 / 17$ & $1 / 9$ & $1 / 19$ & $1 / 12$ & $3 / 24$ & $2 / 16$ & $2 / 19$ & $2 / 10$ \\
\hline Totales & \multicolumn{4}{|c|}{ 7/79 PRE-TEST } & \multicolumn{4}{|c|}{ 6/47 POST-TEST } \\
\hline Otras & $2 / 17$ & $0 / 9$ & $1 / 19$ & $0 / 12$ & $1 / 24$ & $1 / 16$ & $3 / 19$ & $0 / 10$ \\
\hline Totales & \multicolumn{4}{|c|}{ 7/79 PRE-TEST } & \multicolumn{4}{|c|}{ 1/47 POST-TEST } \\
\hline
\end{tabular}


Cuadro 2. Registra la clasificación de concepciones positivistas, transicionales, Lakatosianas y otras, encontradas en el pre y post-test, para la pregunta de investigación realizada a estudiantes del curso "Estructura de la Materia", de la Escuela de Educación, de la Universidad de Los Andes, Venezuela.

\begin{tabular}{|c|c|c|}
\hline SEMESTRE & Pre-test & Post-test \\
\hline \multicolumn{3}{|c|}{ POSITIVISTAS } \\
\hline $\begin{array}{c}\text { SEMESTRE } \\
1\end{array}$ & $\begin{array}{l}\text { No cambian } \\
\text { Cambian por detalles experimentales y } \\
\text { demostraciones } \\
\text { Cambian por fallos experimentales } \\
\text { Por la búsqueda de conocimientos e } \\
\text { investigación } \\
\text { Porque cambian los métodos } \\
\text { Cambian por reformulaciones } \\
\text { Son ciertas y verdaderas }\end{array}$ & $\begin{array}{l}\text { Por los estudios } \\
\text { Por investigaciones } \\
\text { Por experimentos } \\
\text { Por comprobación o demostración } \\
\text { Para conocer de las teorías } \\
\text { Para conocer su veracidad } \\
\text { Las teorías pueden hacerse leyes } \\
\text { Las teorías subsisten a los cambios } \\
\text { Las teorías no son comprobables }\end{array}$ \\
\hline $\begin{array}{c}\text { SEMESTRE } \\
2\end{array}$ & $\begin{array}{l}\text { No cambian } \\
\text { Por investigación } \\
\text { Por nuevos descubrimientos } \\
\text { Por comprobación } \\
\text { Por nuevos métodos } \\
\text { Se estudian para obtener conocimiento } \\
\text { Se estudian para comparar } \\
\text { Son preposiciones consideradas verdaderas } \\
\text { Las teorías son hipótesis }\end{array}$ & $\begin{array}{l}\text { No cambia } \\
\text { Cambian por experimentación } \\
\text { Por descubrimientos } \\
\text { Por demostración } \\
\text { Por la tecnología } \\
\text { Para entender las fallas } \\
\text { Cambian los postulados de la teoría }\end{array}$ \\
\hline $\begin{array}{c}\text { SEMESTRE } \\
3\end{array}$ & $\begin{array}{l}\text { No cambian } \\
\text { No cambian debido a experimentos y } \\
\text { razonamientos } \\
\text { Aprender de ellas para experimentar } \\
\text { Para hacer investigación } \\
\text { Por demostraciones } \\
\text { Por la tecnología }\end{array}$ & $\begin{array}{l}\text { Por verificación } \\
\text { Para generar nuevas teorías } \\
\text { Para saber de ellas y llegar a acuerdos } \\
\text { Para saber su factibilidad } \\
\text { Para desarrollar nuevos experimentos } \\
\text { Unas teorías son sustituidas por otras para llegar } \\
\text { a mayor concordancia con los experimentos } \\
\text { Para desarrollar una teoría se necesitan muchos } \\
\text { años y experimentos } \\
\text { Las leyes de hoy fueron teorías antes } \\
\text { Una teoría es una explicación sobre un conjunto } \\
\text { relacionado de observaciones o experimentos }\end{array}$ \\
\hline $\begin{array}{c}\text { SEMESTRE } \\
4\end{array}$ & $\begin{array}{l}\text { Cambian por comprobación } \\
\text { Por tecnología } \\
\text { Por investigaciones } \\
\text { Por nuevos métodos } \\
\text { Las teorías tienen bases empíricas } \\
\text { Lo que está dicho en la teoría es la única verdad } \\
\text { Las teorías exponen el conocimiento } \\
\text { Las teorías son verificables } \\
\text { De las teorías pueden otras leyes } \\
\text { Las teorías no cambian si están bien planteadas }\end{array}$ & $\begin{array}{l}\text { Por investigaciones } \\
\text { Las estudiamos para entender las modificaciones } \\
\text { Para seguir patrones } \\
\text { Para entender la naturaleza } \\
\text { Para entender conceptos } \\
\text { Las teorías son formas para demostrar el porqué } \\
\text { de los fenómenos físicos, químicos y biológicos } \\
\text { Las teorías son sólo hipótesis muy bien } \\
\text { elaboradas y que cuentan con suficiente } \\
\text { evidencia experimental } \\
\text { Las teorías se sustentan en conceptos que han } \\
\text { sido comprobados con el método científico }\end{array}$ \\
\hline
\end{tabular}


Continuación Cuadro 2.

\begin{tabular}{|c|c|c|}
\hline \multicolumn{3}{|c|}{ TRANSICIONALES } \\
\hline $\begin{array}{l}\text { SEMESTRE } \\
1\end{array}$ & $\begin{array}{l}\text { Son base para estudios posteriores } \\
\text { Por no poseer bases científicas suficientes } \\
\text { Cambian porque no son absolutas } \\
\text { Son una forma abstracta de la ciencia }\end{array}$ & $\begin{array}{l}\text { Por estar incompletas } \\
\text { Para entender el cambio } \\
\text { Para entender los nuevos conocimientos } \\
\text { Las teorías son relativas } \\
\text { Las teorías son supuestos }\end{array}$ \\
\hline $\begin{array}{l}\text { SEMESTRE } \\
4\end{array}$ & $\begin{array}{l}\text { Por evolución } \\
\text { Para mejorar el conocimiento }\end{array}$ & $\begin{array}{l}\text { No cambian, son sustituidas } \\
\text { Por evolución }\end{array}$ \\
\hline \multicolumn{3}{|c|}{ LAKATOSIANAS } \\
\hline $\begin{array}{l}\text { SEMESTRE } \\
1\end{array}$ & $\begin{array}{l}\text { Cambian por evolución } \\
\text { Por contradicción entre teorías }\end{array}$ & Por refutación \\
\hline \multicolumn{3}{|c|}{ OTRAS } \\
\hline $\begin{array}{l}\text { SEMESTRE } \\
1\end{array}$ & $\begin{array}{l}\text { Cambian poco } \\
\text { Son formas de explicar el conocimiento }\end{array}$ & ------------ \\
\hline $\begin{array}{l}\text { SEMESTRE } \\
2\end{array}$ & se presentaron varios modelos & ----------- \\
\hline $\begin{array}{l}\text { SEMESTRE } \\
3\end{array}$ & $\begin{array}{l}\text { Aprender de las teorías porque enseñan de una } \\
\text { manera monótona y al caletre }\end{array}$ & $\begin{array}{l}\text { Las teorías son formas de ver desde el punto de } \\
\text { vista de cada científico }\end{array}$ \\
\hline $\begin{array}{l}\text { SEMESTRE } \\
4\end{array}$ & $\begin{array}{l}\text { Por influencia de personas ilustradas } \\
\text { Se estudian porque sirven de base cognitiva } \\
\text { Crear nuevas teorías pero sin obviar las } \\
\text { anteriores }\end{array}$ & ------------- \\
\hline
\end{tabular}

que una teoría axiomatizada tenga múltiples ventajas, como un conjunto de conceptos (fundamentales y derivados) sistematizados, coincidencia con hechos empíricos, disminución de ambigüedades, hipótesis bien expresadas, predicciones y pruebas de generalidad. Esta tendencia también coincide con la idea de Kritsonis (2005), quien afirma que el cambio 
en las teorías también viene dado por la idea que la nueva teoría desarrollada juega un mejor papel en la explicación y en la comprensión de hechos asociados a ella. Por tanto, la nueva teoría tiende a afianzarse en función de un nuevo marco explicativo, que permite no solo explicar mejor lo conocido, sino intentar predecir mejor los hechos científicos.

Pero, además, en este mismo marco, algunos llegan a creer que las teorías no cambian, dado que son preposiciones consideradas verdaderas, lo cual, les confiere la propiedad de ser una referencia de estudio o quizás les confiera un carácter de dogma científico, lo que se puede entender como un grado superlativo de la sistematización teórica, un claro aporte del positivismo.

Aparecieron algunas concepciones transicionales, como la idea del cambio por nuevos conocimientos que, gradualmente, van sustituyendo a los anteriores por evolución o porque las teorías no son absolutas y cada etapa se marca por la presencia de un conocimiento científico no afianzado suficientemente, que debe ser revisado, lo que tiende a corresponderse con algunas visiones de la ciencia que transcienden la formalización y en las que se ofrece una perspectiva diferente, donde las teorías son consideradas una estructura dinámica y cambiante en sus entidades de crecimiento, como los planteamientos Kuhneanos, desde el estructuralismo científico y Lakatosinanos, desde la disidencia progresiva.

No pareció ser importante responder cuál es el motivo de estudiar las teorías, admitiéndose, lacónicamente, que pueden ser la base para estudios posteriores, pero sin mayores explicaciones al respecto. Esto parece ser un aspecto cercano a la falta de aprendizaje histórico en la ciencia, pues, hoy, se reconoce que la perspectiva histórica nos acerca a una ciencia visualizada como actividad humana, no endiosada que, en ocasiones, se hace penosamente zigzagueante y, en otras, con carácter falible, capaz de corregir sus errores, aproximándose a los procesos y relevantes personalidades, que han ayudado a impulsar sus bases de saber durante muchos siglos y por motivaciones distintas (Brey, 2014). Así, se entiende que la enseñanza de la ciencia planteada sin la dimensión de su historia no encuentra importancia al estudio de las teorías desde su desarrollo, sino solamente desde sus aplicaciones, tentativa, quizás, un tanto afianzada en la expectación empírica Baconeana y Lockeana, donde el hincapié recae en la experiencia de los sentidos para la búsqueda del conocimiento y no en la especulación intuitiva o la deducción de las explicaciones.

Otras expresiones Lakatoseanas expusieron el cambio por reformulaciones o por contradicción entre teorías, lo que puede poner en evidencia la idea de un posible conflicto entre campos de pensamiento que rivalizan en el avance de la ciencia, como producto de un proceso perpetuo de refutación o reforzamiento, que resulta de enfrentar programas de investigación que siguen líneas diferentes, dado sus rasgos de comprometer el pensamiento en corrientes que se enfrentan y requieren de la aparición de evidencia, para poder refutarla o aceptarla en el plano de los contextos de trabajo que involucran la presencia de teorías rivales que están en un paradigma o que cambian, debido a los paradigmas emergentes.

Esto último parece coincidir con la relevancia que ha cobrado el estudio de las disidencias en la enseñanza de las ciencias, donde se estila que las metas generales de la educación científica contemplen a los estudiantes, desarrollando procesos de construcción del conocimiento, donde se conciba la ciencia como una actividad racional tentativa y sujeta a posibles reformulaciones, aunque se sabe que el éxito es limitado, ya que las ideas inadecuadas sobre la ciencia, se mantienen vigentes (Campanario, 2004). Quizás por ello, conquistar adeptos a concepciones dinámicas sobre la estructura del conocimiento científico, sea una tarea del día a día.

Igualmente, se encontró que, en cuanto a los ejemplos mencionados, se observa una variedad que, en ningún caso, expone un análisis completo o parcial sobre las posturas de los cambios, sino que se limitan a ejemplificar algunas teorías, como la atómica o la de Darwin, pero sin hacer alusiones a sus transformaciones históricas; este es un factor que quizás se pueda entender como determinante, ya que desde la Filosofía de la Ciencia tiende a rebatirse que las motivaciones personales y la realidad del trabajo diario de los investigadores puede ser crucial en la construcción del conocimiento, es decir, tiende darse un papel prep.0onderante a la objetividad (Alvarado \& Carrillo, 2009), pero ninguno hace un ejemplo concreto sobre un cambio en una teoría conocida de la ciencia o algún paradigma sobre una teoría en la química que no sea la teoría atómica, mencionada en el enunciado de la pregunta, lo que se puede entender como una falta de profundización en el conocimiento epistémico de las teorías.

Como se evidencia en el cuadro 3 y teniendo como referencia los cuadros 1 y 2 , durante las observaciones, se notó una estructura similar. Se admite, bajo una posición positivista, que las teorías cambian, porque son parte del pensamiento de cada época; sin embargo, se afirma que no todas las teorías pueden cambiar, por cuanto hay poderes que no les conviene que cambien. Esta postrema idea es de especial interés, puesto que se ha discutido decididamente el enfoque, sobre el cual, la reputación de los investigadores es trascendental, por tanto, la notoriedad académica parece importante en el camino de facilitar la aceptación de nuevas ideas o mantener las ya existentes en el contexto científico (Campanario \& Martin, 2004). En todo caso, la tendencia más común encontrada es que para que una teoría cambie 
Cuadro 3. Registra/resume la clasificación de tendencias positivistas y transicionales, reconocidas en el proceso de observación, durante los periodos de investigación en los estudiantes del curso "Estructura de la Materia", de la Escuela de Educación, de la Universidad de Los Andes, Venezuela.

\begin{tabular}{|l|}
\multicolumn{1}{|c|}{ Tendencia POSITIVISTA- Indicadores } \\
\hline Las teorías tienen que cambiar porque las sociedades cambian \\
La teoría cambia por datos y leyes \\
Las teorías son anteriores a las leyes \\
Las teorías se convierten en leyes \\
Las teorías se formulan sobre la base de la observación de regularidades \\
Las teorías no son tan importantes como las leyes \\
Las teorías describen y no explican porque forman parte de la imaginación \\
Las teorías son esencialmente falsas pues tienen que ser demostradas \\
Las teorías cambian en sus postulados, pero no en su estructura. \\
Las teorías cambian en la medida que aparecen nuevas leyes \\
Los intereses de algunos científicos pueden tener peso en su apreciación \\
El uso de buenos instrumentos de medición elimina la apreciación personal \\
El papel de la experimentación fue fundamental \\
La frase "a ciencia cierta" es asociada a la regularidad de la ciencia \\
Una teoría es un esquema conceptual más amplio que el de las leyes \\
\hline \multicolumn{1}{c|}{ Tendencia TRANSICIONAL - Indicadores } \\
\hline Las teorías son un conocimiento contingente \\
La teoría atómica es cambiante para mejorarse en término de sus postulados \\
El camino para modificar y mejorar el entendimiento está dado por la tecnología \\
La teoría atómica ha tenido la posibilidad de cambiar al mejorar sus postulados. \\
Las leyes no cambian y las teorías en general no cambian tampoco, sólo se mejoran. \\
La teórica se modifica por sustitución o evolución \\
\hline
\end{tabular}

tienen que haber datos y leyes que las cambien: idea positivista. Consideración anterior, que compagina con la idea, sobre la cual, la mayor parte de las personas concibe a la ciencia como un conocimiento correcto, obtenido mediante un sistema infalible (Campanario, 2004). Por ello, resulta perentorio cuestionar tales concepciones tratando acercar al estudiantado a los procesos históricos y haciendo una mejor valoración de los conocimientos disidentes.

A la par, la teoría es una ayuda para captar la imagen completa del mundo físico, siendo un esquema conceptual y teórico para exponer los fenómenos que se observan (Rizo, 2014). Nótese así, que la teoría tendría a entenderse como un nivel más general, cuyas aplicaciones descriptivas y explicativas se sustentan en regularidades, condensadas en leyes.

Durante los debates de laboratorio afloró que la teoría atómica es cambiante para mejorarse en término de sus postulados, pero sabiendo que ella sigue siendo la misma, es decir, ha cambiado en sus postulados, pero no en su estructura (Doménech et al. 2013). Tal extracción de las concepciones estudiantiles puede corresponder a la idea de Carnap, sobre la cual, se reconoce a las teorías materialistas, como aquellas que dan cuenta de fenómenos empíricos gobernados por ciertas leyes, directamente accesibles a la observación; de este modo, parece admitirse, que la teoría permite avanzar en la idealización de un conocimiento y la ley constituye la base del saber científico.

Para los estudiantes parece claro que las leyes permiten elaborar acciones concretas, como la tabla periódica y que son ideas generales de cómo se entiende un objeto o fenómeno. Tal trazado parece sustentarse en la tentativa que la ciencia se distingue de otros procesos de conocer, mediante el uso de estándares empíricos, evidencias lógicas y actitud escéptica (Garritz, 2006). Por ello, el uso de buenos instrumentos de medición elimina la "apreciación personal", permitiendo buenos experimentos y conclusiones más correctas. Es así, como el grueso del estudiantado admite que las conclusiones deben responder a hechos verificables, pues de lo contrario, los resultados de una investigación quedan referenciados a quienes hacen las investigaciones y no al fenómeno estudiado. 
La idea generalizada es que las leyes no cambian y las teorías en general no cambian tampoco, solo se mejoran. Acá, nuevamente, se aporta evidencia en que los estudiantes tienen a una visión inductivista, desde el empirismo y el positivismo (Rodríguez \& López, 2006). Se asume, entonces, que los experimentos, mediante la tecnología, generaron nuevas ideas, que al ser estudiadas de forma sistemática, derivaron en leyes. Algunos denominan a esta tendencia el "experimentalismo crédulo", donde se tiene, por cierto, que la experimentación hace posible la verificación indiscutible de los postulados (Cutrera, 2006). Por ello, parece asumirse que el papel de la experimentación fue fundamental para conocer la estructura y el funcionamiento del átomo, destacándose, nuevamente, la influencia del llamado empirismo lógico. Todo esto hace pensar en unas fuertes ideas que han sido formadas desde el bachillerato y que el solo hecho de trabajar en el laboratorio, realizar experiencias y discutir arduamente la temática, no las hace cambiar a priori.

En el ámbito de los debates, por medio de presentaciones, se admite que las teorías solo describen de forma general; para conocer los procesos, realmente hacen falta estudios desde la experimentación y la verificación de cómo funcionan, mediante las leyes de la naturaleza, aspecto que se ajusta a la conocida racionalidad instrumental, con su idealismo e individualismo (Sierra, 2006). Las tendencias observadas nos deben hacer reflexionar sobre el papel del docente, la acción participativa estudiantil y, sobre todo, en los métodos didácticos usados para introducir y desarrollar una didáctica de las ciencias más cercana a la humanidad y a los fenómenos naturales. Esto es especialmente cierto, de acuerdo con lo esbozado por Gomez-Zweip (2008), al referirse a las formas de enseñar en franca dinámica con los intereses y las necesidades del estudiantado; esto quiere decir, que inclinarnos hacia las necesidades de los estudiantes, no solo es un buen aporte para el desarrollo de sus conocimientos, sino que, además, allana el camino que se debe seguir para la reformulación de cursos en pro de mejorar la enseñanza de las ciencias y de sus concepciones históricas y metodológicas.

Parecen existir dos procesos de modificación teórica: sustitución y evolución. Ciertamente, este último punto, se introduce en el quicio de las tendencias actuales de asumir la ciencia, donde las concepciones sistémicas y evolucionista parecen jugar un papel de suma importancia para el desarrollo de la ciencia y su didáctica (Padrón, 2013). Y es que mientras las posiciones epistemológicas asumen que la realidad es cognoscible, se entiende que el mismo cognoscitivismo humano es consecuencia de la evolución del pensamiento durante la interacción con la realidad; condiciones muy interesantes, para entender que la didáctica de la ciencia progresista debe abarcar todo lo que pueda en términos de las diversas posiciones históricas de los científicos, en torno a un conocimiento. Casualmente, esto se asemeja a la propo- sición de Clement (2008), quien nos hace ver que los modelos de explicación son fundamentales para generar interés en los estudiantes y, por tanto, en las propuestas de trabajo, es decir, en la pretensión de entender la ciencia como un conocimiento cambiante. Por tal razón, asumir el cambio en los cursos es la primera posible casusa de éxito en mejorar la enseñanza, dado que los modelos de explicación desde una ciencia "menos racional" y "más humana", se hace más atractiva para el estudiantado, que suele ver a la ciencia como algo finamente maquinado, para ser una tranca en los procesos de formación del conocimiento.

Se admite que el teoricismo aporta ideas generales para el debate abierto en la ciencia, pero el experimentalismo constituye las bases del verdadero saber científico. Entonces, el teoricismo se puede entender, en la idea Althussereana, sobre la cual el teoricismo está signado por el prelado de la teoría sobre la práctica; no obstante, en la otra acera, el experimentalismo o realismo lleva a suponer que la intervención para la búsqueda del conocimiento requiere el abordaje de la realidad de los problemas desde el uso de suficientes herramientas técnicas y con conocimiento efectivo de las cosas (Grassi, 2007). Como vemos, aparecen posiciones aparentemente contrapuestas que en seno del trabajo científico deben encontrar las suficientes ataduras, que permitan la emergencia de tanta teoría y tanta práctica para la búsqueda del conocimiento. Y esa búsqueda del conocimiento tiene que ver, según Özdemir \& Clark (2007), con la aceptación de nuevos estudios sustentados en atributos más fuertes, que van apareciendo en la medida que las visiones de la ciencia cambia y las metodologías se renuevan. Siento esto especialmente importante, por cuanto recalca el hecho de las nuevas visiones del mundo, de las nuevas perspectivas que aparecen en cada época, como caldo de cultivo, para la avanzada del conocimiento.

La frase "a ciencia cierta" es asociada con la regularidad de la ciencia expresada en las leyes y no con el conocimiento teórico finamente elaborado en las teorías, concepción muy cercana a la ciencia mecanicista de causa-efecto. Estos aspectos son significativos, por cuanto parecen muy arraigados en el estudiantado y difíciles de ser superados en un curso; si bien deba reconocerse, algunos migran sus concepciones de forma progresiva.

Se observó que los estudiantes tienden a diferenciar leyes y teorías, tanto por su origen como por su desarrollo y fines. Tal consideración, se puede incluir en el llamado período clásico de las teorías científicas, donde las teorías son sistemas axiomáticos de interpretación empírica, cuyo estudio supone albergar una estructura estática, que puede cambiar solo a la luz de la nueva evidencia experimental (Bermúdez, 2015). Nótese, que las teorías y las leyes son en verdad dos símbolos de una misma carta, que concibe al mundo natural como 
algo producto de la observación. Se entiende, pues, que aún en medio de un océano de ideas ligadas a un conocimiento provisional, siempre germina la semilla de un esquema modal muy perfilado, en lo que proporciona más confianza al humano común, sus sentidos.

Parece evidenciarse que los docentes en formación abordan su aprendizaje desde una visión epistemológica absolutista (Guisásola \& Morentin, 2007). Ciertamente, las tentativas positivistas son el trozo más grande del pastel y apuntan hacia una educación de las ciencias, que debe ser reformada en aras de mirar otras opciones del pensamiento científico. Así, las concepciones de los futuros docentes, se mantienen ancladas en una idea de ciencia racional, dogmática (Ravanal \& Quintanilla, 2010). Algunas expresiones sobre las teorías, las resaltan como formas para demostrar el por qué de los fenómenos y se entiende a la demostración, como el eje fundamental de trabajo. Esto, se puede asociar a la idea expuesta por Barseghyan (2012), quien hace ver que la teoría es una estructura generada de un proceso general, que puede cambiar en función de los aportes de las leyes a las explicaciones científicas, es decir, las nuevas demostraciones. Siendo esta tendencia positivista y que es una de las guías principales de los planteamientos de la ciencia actual y su enseñanza, donde poco se suele debatir el papel del pensamiento como precedente generado, tanto de la aparición de las leyes como de las teorías.
Otras concepciones transicionales orbitan en torno a que tal posibilidad de cambio se genere, dado que las teorías estén incompletas, en el entendido que una teoría no deja de cambiar hasta que no responda todas las preguntas. Tales percepciones coinciden con algunos trabajos donde se ha hallado que, a nivel universitario, las concepciones se caracterizan por una búsqueda de paradigmas renovados (Alvarado \& Carrillo, 2009), siendo este un aspecto de suma importancia, por cuanto la idea de concepciones emergentes es de un gran valor para la trasformación didáctica. Así, debemos decir que, como se puede observar en la figura 1 , en el esquema de nuestro diseño del curso, concebimos al conocimiento científico como un encuentro de oportunidades didácticas, mediadas por cinco dimensiones, donde lo práctico, lo teórico, lo lúdico, lo recreativo y lo creativo, se funden en un proceso, para el descubrir, el inventar, el comprender y el proponer, como mecanismo de participación y acción de trabajo.

Algunas otras expresiones fueron de corte Lakatosiano, dado que exponen que una teoría debe cambiar por no ser correcta cuya verificación se presenta o se puede dar por refutación con las teorías rivales. Tal plataforma, se puede ubicar en el reconocido período historicista, donde se asume a la ciencia como un proyecto de investigación ligado a la historia; aspecto interesante, para la valoración de nuestro trabajo, por dar cuenta de unas miradas diversas de los procesos de la ciencia y sus contextos.

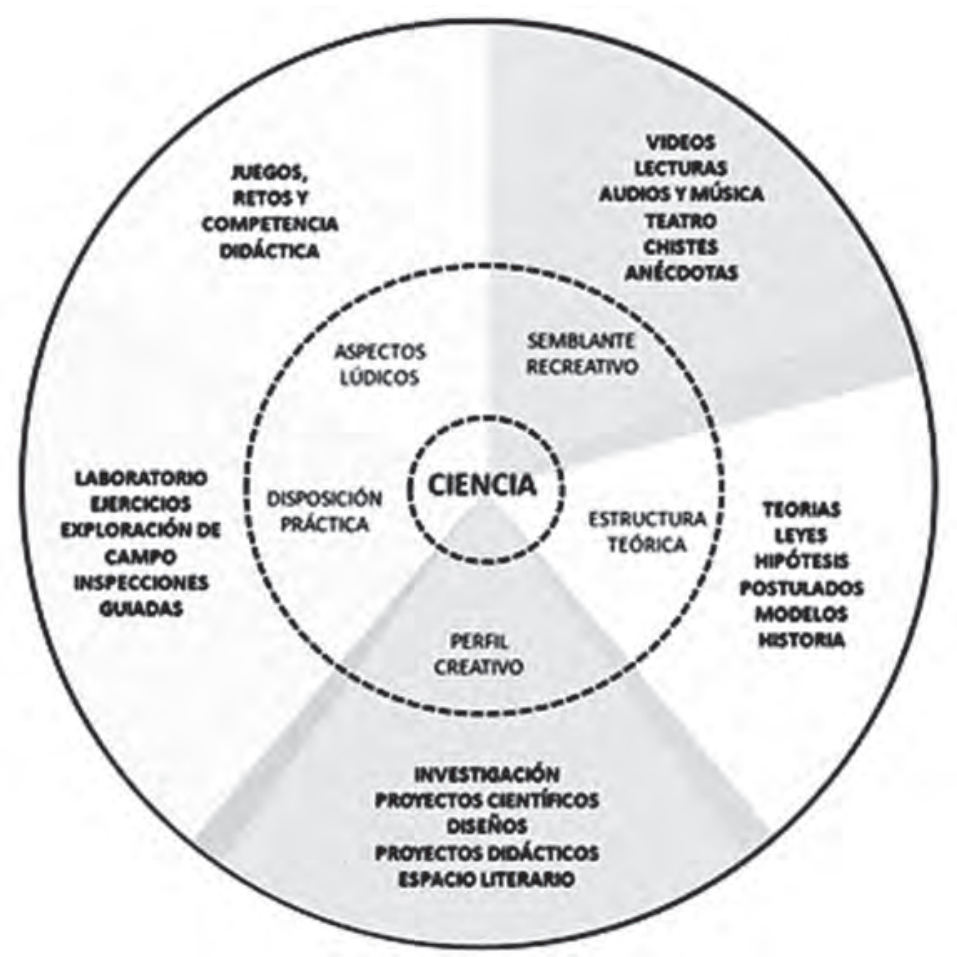

Figura 1. Representación de los perfiles usados, para la definición de acciones didácticas, que permitieron el desarrollo del curso "Estructura de la Materia", en la Escuela de Educación, de la Universidad de Los Andes, Venezuela. 
Finalmente, conviene mencionar la frecuencia de ejemplificación de teorías dada por los grupos, donde se destacan: teoría de evolución (8), teoría atómica (3), creacionista, de generación espontánea, de la tabla periódica, forma de la tierra (redonda), la teoría geocéntrica, la teoría heliocéntrica, teoría del flogisto, teoría la relatividad y teoría de números. Notándose, primero, el gran impacto que parece tener la teoría de la evolución en el aprendizaje científico (Bermúdez, 2015) y, en segundo lugar, el hecho, sobre el cual, las argumentaciones sobre el cambio en las teorías llega desde concepciones positivistas, desde la experimentación y sin importar mucho el camino seguido para el cambio en una teoría, aseveraciones, que concuerdan con algunos trabajos, que hacen ver ciertas estrategias y muestran ser significativas para las ideas de los estudiantes (López et al. 2013).

Se concluye, que hubo influencia del curso "Estructura de la Materia", influencia expresada en una variación de concepciones desde el Positivismo hacia otras posiciones, tanto Transicionales como Lakatosianas. Las concepciones epistemológicas en estudiantes que arribaron al curso mostraron un enorme dominio del Positivismo. Durante el proceso de observación en el curso, se evidenció el domino del Positivista, aunque se notó un cambio gradual a concepciones alternativas hacia el final del curso. Los factores metodológicos más útiles para el cambio en las concepciones fueron las clases apuntaladas en video.

La tendencia inicial para diferenciar teorías y leyes fue el carácter conjetural, de las primeras y lo normativo, de las segundas, en un marco experimental. Al final del curso, la disposición para reconocer teorías científicas tendió a migrar ligeramente hacia posiciones más críticas, fundamentalmente, a ideas Transicionales. Las participaciones libres de los estudiantes aumentaron con el correr del semestre, lo que se puede entender como ganancia de confianza de los estudiantes en los planteamientos del curso. Las posturas Positivistas mostraron ser suficientemente fuertes, como para ser estimadas concepciones nativas en el proceso educativo, para el reconocimiento de teorías científicas. Las concepciones no clasificadas tendieron a la reducción, lo que se puede percibir como un afianzamiento de la estructura epistemológica estudiantil. La teoría más citada, ejemplificada y conceptualizada fue la "Teoría sobre la Evolución", lo que hace ver la fuerza discursiva y el impacto de esta teoría en el conocimiento general.

Agradecimientos: Al Doctorado en Ciencias de la Educación, por el apoyo institucional prestado. Al Consejo de Desarrollo Científico, Humanístico y Tecnológico, por el financiamiento de esta investigación. Conflicto de intereses: Hacemos constar que este manuscrito fue desarrollado con la intervención de sus autores, quienes declaramos la no existencia de conflicto de intereses que ponga en riesgo la validez de los resultados presentados.

\section{BIBLIOGRAFÍA}

1. ALVARADO, M.; CARRILLO, L. 2009. Concepciones de ciencia en la UNAM: el impacto en la educación universitaria. Educación, ciencia y tecnología. México D.F. (México). Ediciones UNAM, 11p.

2. BARSEGHYAN, H. 2012. A Theory of Scientific Change. Institute for the History and Philosophy of Science and Technology, University of Toronto (USA).318p.

3. BERMÚDEZ, G. 2015. Los orígenes de la Biología como ciencia. El impacto de las teorías de evolución y las problemáticas asociadas a su enseñanza y aprendizaje. Rev. Eureka (España). 12(1):66-90.

4. BREY, J. 2014. El fraude en la investigación científica. Real Academia Sevillana de Ciencias. Sevilla (España): Universidad de Sevilla. 11p.

5. CAMPANARIO, J. 2004. Científicos que cuestionan los paradigmas dominantes: algunas implicaciones para la enseñanza de las ciencias. Rev. Electr. Enseñanza Ciencias (España). 3(3):53-68.

6. CAMPANARIO, J.; MARTIN, B. 2004. Challenging current Physics paradigms. J. Scientific Explor. (USA). 18(3):421-438.

7. CLEMENT, J. 2008. The role of explanatory models in teaching for conceptual change. In: Vosniadou, S. (ed.), International handbook of research on conceptual change (USA). p.417-452.

8. CUTRERA, G. 2006. La actividad científica y la génesis del conocimiento científico en los textos escolares de ciencias naturales: un análisis de clasificación. Rev. Iberoam. Educación (España). 42:98-117.

9. DOMÉNECH, J.; SAVALL, F.; MARTÍNEZ, J. 2013. ¿̇Los libros de texto de bachillerato Introducen adecuadamente los modelos atómicos de Thomson y Rutherford? Enseñanza ciencias, Rev. Investig. experiencias didácticas (España). 31(1):29-43.

10. FOUREZ, G. 2008. Cómo se elabora el conocimiento. La epistemología desde un enfoque socio-constructivista. Narcea, España.188p.

11. GARRITZ, A. 2006. Naturaleza de la ciencia e indagación: cuestiones fundamentales para la educación científica del ciudadano. Rev. Iberoam. Educación (España). 42:127-152. 
12. GRASSI, E. 2007. Problemas de realismo y teoricismo en la investigación social y en el Trabajo Social. Rev. Katál Florianópolis. 10:26-36.

13. GOMEZ-ZWEIP, S. 2008. Elementary teachers' understanding of students' science misconceptions: Implications for practice and teacher education. J. Sci. Teacher Education (USA). 19:437-454.

14. GUISÁSOLA, J.; MORENTIN, M. 2007. ¿Comprenden la naturaleza de la ciencia los futuros maestros y maestras de Educación Primaria? Rev. Electr. Enseñanza Ciencias. 6(2):246-262.

15. KRITSONIS, A. 2005. Comparison of change theories. Int. J. Scholarly Academic Intellectual Diversity (USA). 1:1-7.

16. LÓPEZ, W.; MOLINA, L.; CÁRDENAS, M.; BIANCHI, G.; QUINTERO, H.; ESCALONA J. 2013. Un estudio de la relación entre las concepciones de aprendizaje, las estrategias metacognitivas y el rendimiento académico en cursos de química universitaria. Enseñanza de las Ciencias (España), Número Extra. X:19661971.

17. ÖZDEMIR, G.; CLARK, D. 2007. An Overview of Conceptual Change Theories. Eurasia J. Math., Science \& Technology Education (Turkey). 3(4):351-361.

18. PADRÓN, J. 2013. Epistemología evolucionista: una visión integral. Bogotá, (Colombia), Ediciones EntreTemas. 123p.

19. PONTES, A.; POYATO, F.; OLIVA, J. 2015. Concepciones sobre el aprendizaje en estudiantes del máster de profesorado de educación secundaria del área de ciencia y tecnología. Profesorado: rev. curriculum y formación del profesorado (España). 19(2):225-243.

20. RAVANAL, E.; QUINTANILLA, M. 2010. Caracterización de las concepciones epistemológicas del profesora- do de biología en ejercicio sobre la naturaleza de la ciencia. Rev. Electr. Enseñanza Ciencias. 9(1):111124.

21. RESTREPO, J. 2014. Concepción y prácticas de aula del maestro de ciencias naturales que promueven las relaciones CTS. Universidad de Antioquia, Antioquia (Colombia): Ediciones Antioquia. 146p.

22. RIZO, M. 2014. El papel de las teorías de la comunicación en la construcción del campo académico de la comunicación. Reflexiones desde la historia, la epistemología y la pedagogía. Correspondencias \& Análisis (Perú). 4:239-258.

23. RODRÍGUEZ, D.; LÓPEZ, A. 2006. ¿Cómo se articulan las concepciones epistemológicas y de aprendizaje con la práctica docente en el aula? Tres estudios de caso de profesores de secundaria. Rev. Invest. Educativa (México). 9(31):699-719.

24. SCHETTINI, P.; CORTAZZO, C. 2015. Análisis de datos cualitativos en la investigación social: procedimientos y herramientas para la interpretación de información cualitativa. Universidad Nacional de La Plata, La Plata (Argentina): Ediciones UNP. 119p.

25. SERRANO, S. 2015. Lenguaje y ciencia: percepciones del profesorado sobre el lenguaje en la construcción del conocimiento científico. Educere (Venezuela). 19(63):537-548.

26. SIERRA, C. 2006. La reproducción de experimentos históricos en relación con la forja de ethos científico. Rev. Eureka para Enseñanza y Divulgación Científica (España). 3(1): 60-76.

Recibido: Noviembre 18 de 2015

Aceptado: Septiembre 30 de 2016

\section{Cómo citar:}

Escalona Tapia, J.; Fontal Rivera, B. 2016. Cambio en las teorías científicas desde la visión de estudiantes de educación en la Universidad de los Andes, Venezuela. Rev. U.D.C.A Act. \& Div. Cient. 19(2): 467-477. 